\title{
BIBLIOGRAPHY
}

1. A. P. Morse, The behavior of a function on its critical set, Ann. of Math. 40 (1939), 62-70.

2. Arthur Sard, The measure of the critical values of differentiable maps, Bull. Amer. Math. Soc. 48 (1942), 883-890.

3. H. Whitney, $A$ function not constant on a connected set of its critical points, Duke Math. J. 1 (1935), 514-517.

Instituto de Mathematica, Pura e Aplicada, Rio de Janeiro, Brazil

\section{THE DEGREE OF APPROXIMATION BY LINEAR OPERATORS}

\author{
PHILIP C. CURTIS, JR. ${ }^{1}$
}

I. Introduction. In 1959 Berman [1] observed the following: If $T$ denotes the unit circle, $C(T)$ the continuous functions on $T$, and $E_{n}(f)$ the error in the best approximation in the sup norm to $f$ by a trigonometric polynomial of order $n$, then there could not exist a sequence of linear operators $T_{n}$ mapping $C(T)$ into the trigonometric polynomials of order $n$, which satisfied

$$
\left\|f-T_{n} f\right\| \leqq K E_{n}(f)
$$

for some fixed constant $K$ and $f \in C(T)$. The reason for this is easy to see. Suppose (1) is satisfied for some sequence $\left\{T_{n}\right\}$ and all $f \in C(T)$. If $\Pi_{n}$ denotes the space of trigonometric polynomials of order $\leqq n$, then for $f \in \Pi_{n}, E_{n}(f)=0$. Hence, $T_{n} f=f$ or $T_{n}^{2}=T_{n}$. But by a theorem of Nikolaev [3, p. 494], $\left\|T_{n}\right\| \geqq K \log n$. Since $E_{n}(f) \rightarrow 0$, this is a contradiction. A similar observation holds for $L_{1}(T)$ where $E_{n}(f)$ is the best approximation by a trigonometric polynomial of order $n$ to $f$ in the $L_{1}$ norm.

In this note I would like to elaborate on this observation of Berman's and make some applications to Fourier series which appear to be new. Let $T_{n}$ be a sequence of bounded linear operators from $C(T)$ into $\Pi_{n}$. In place of $E_{n}(f)$ let $D_{n}(f)$ be any continuous mapping from $C(T)$ to the non-negative reals which vanishes on $\Pi_{n}$. In addition to the case $D_{n}(f)=E_{n}(f)$ we may take $D_{n}(f)=\left\|f-P_{n} f\right\|$ where $P_{n}$ is a bounded projection of $C(T)$ onto $\Pi_{n}$. Then, if $T_{n}^{2} \neq T_{n}$ for each $n$, it

Received by the editors February 20, 1964.

1 The research reported here was supported by TRW Space Technology Laboratories and by the National Science Foundation. 
is never true that $\left\|f-T_{n} f\right\|=O\left(D_{n}(f)\right)$ for all $f$. This is still true even if $\sup _{n} D_{n}(f)=\infty$ for some $f$. Specifically the set of functions $f \in C(T)$ for which there exist constants $n_{f}, k_{f}$ depending on $f$ for which

$$
\left\|f-T_{n} f\right\| \leqq k_{f} D_{n}(f), \quad n \geqq n_{f},
$$

is of the first category in $C(T)$. An interesting consequence of this lemma is obtained by taking $D_{n}(f)=\left\|f-S_{n} f\right\|, S_{n} f$ the $n$th partial sum of the Fourier series and $T_{n} f=\sigma_{n} f=\left(S_{1} f+\cdots+S_{n} f\right) / n$. Then of course, $\left\|f-\sigma_{n} f\right\| \rightarrow 0$, and, therefore, the set of functions $f$ such that no sequence $S_{n_{k}} f \rightarrow f$ uniformly on $T$ is a set of the first category in $C(T)$. At first glance this appears mildly astonishing since those $f$ for which $\sup _{n}\left\|S_{n} f\right\|=\infty$ are of the second category. This set of the first category is not empty, however, since in 1944 Menshov [2] constructed an example of a continuous function $f$ such that for each sequence $S_{n_{k}} f$ there exists a point $x \in T$ such that $\left(S_{n_{k}} f\right)(x)$ diverges at $x$.

The observations concerning norm convergence hold verbatim in $L_{1}$. Also $\left\{f \in L_{1}: \lim \inf _{n}\left\|f-S_{n} f\right\|_{1}>0\right\}$ is not empty. For if $\left\{a_{n}\right\}$ is convex, and $a_{n} \rightarrow 0$, then $\frac{1}{2} a_{0}+\sum_{n=1}^{\infty} a_{n} \cos n x$ defines a function in $L_{1}$ for which $\left\|f-S_{n} f\right\|_{1}=\pi a_{n}\left\|S_{n}\right\|+o(1)$. Thus $\lim _{n}\left\|S_{n} f\right\|_{1}$ may be infinite. (See $[4$, pp. 182-185] for details.)

II. The following elementary result is the key to these observations.

Lemma. Let $E$ be a Banach space, $\left\{E_{n}\right\}$ a sequence of closed subspaces satisfying $E_{n} \subset E_{n+1}$ and $\mathrm{ClU} E_{n}=E$. For each $n$ let $T_{n}$ be a bounded linear operator mapping $E$ into $E_{n}$ and let $D_{n}$ be a continuous function mapping $E$ into the non-negative reals which vanishes on $E_{n}$. Let $F_{k, n} \equiv\left\{x:\left\|x-T_{m} x\right\| \leqq k D_{m}(x), m \geqq n\right\}$. Then $T_{k, n}$ is a closed set, and if $F_{k, n}$, contains an open set it follows that for some integer $m_{0}$, $T_{m}^{2}=T_{m}$ for $m \geqq m_{0}$.

Proof. In the applications we usually have either $D_{n}(x)=\left\|x-P_{n} x\right\|$ where $P_{n}$ is a projection of $E$ onto $E_{n}$ or $D_{n}(x)=\inf _{y \in E_{n}}\|x-y\|$. Suppose there exists an $x_{0} \in E, \delta>0$, such that $\left\{x:\left\|x-x_{0}\right\|<\delta\right\} \subset F_{k, n}$. By the density of $U_{j} E_{j}$ we may assume $x_{0} \in E_{m_{0}}$ for some $m_{0} \geqq n$. For $m \geqq m_{0}$, if $x \in E_{m}$ and $\|x\|<\delta$, then $x+x_{0} \in F_{k, n}$ and

$$
\left\|x+x_{0}-T_{m}\left(x+x_{0}\right)\right\| \leqq k D_{m}\left(x+x_{0}\right)=0 \text {. }
$$

Therefore, $T_{m} x=x$, and by the homogeneity of $T_{m}, T_{m} x=x$ for all $x \in E_{m}$, or $T_{m}^{2}=T_{m}$.

CoROLlaRY. Under the same assumptions if $T_{m}^{2} \neq T_{m}$ for each $m$, then 
$F=\left\{x \in E\right.$ : there exist constants $n_{x}, k_{x}$ for which $\left\|x-T_{m} x\right\| \leqq k_{x} D_{m}(x)$, $\left.m \geqq n_{x}\right\}$ is of the first category in $E$.

Proof. $F=U F_{k, n}, k, n=1,2, \cdots$.

The observations concerning Fourier series are special cases of the following

CoRollary. If $T_{m} x \rightarrow x$ for each $x$ and $T_{m}^{2} \neq T_{m}$ for each $m$ then $\left\{x: \lim \inf _{m} D_{m}(x)>0\right\}$ is of the first category in $E$.

The lemma, together with Nikolaev's theorem and the uniform boundedness principle, yields a sharper form of Berman's theorem.

CoROLlaRy. Let $T_{m}$ be bounded linear operators mapping $C(T)$ into $\Pi_{m}$. Then $\left\{f:\left\|f-T_{m} f\right\| \leqq k_{f} E_{m}(f), m \geqq n_{f}\right\}$ is of the first category in $C(T)$.

If $T_{m}$ is the Fejer operator $\sigma_{m}$, then there are nonconstant functions with this property. For by a classical theorem of Bernstein's $[3, \mathrm{p}$. 99] if $\alpha_{m} \downarrow 0$, there exists an $f \in C(T)$ such that $E_{m}(f)=\alpha_{m}$. But if $\alpha_{m}=1 / m^{\alpha}, 0<\alpha \leqq 1$, then for such $f$,

$$
\left\|\sigma_{m} f-f\right\| \leqq \frac{k}{m^{\alpha}}
$$

cf. [4, pp. 120-123].

\section{BIBLIOGRAPHY}

1. D. L. Berman, On the impossibility of constructing a linear polynomial operator giving an approximation of best order, Uspehi. Mat. Nauk. 14 (1959), 141-142.

2. D. Menshov, Sur les sommes partielles des séries de Fourier des fonctions continues, Mat. Sb. 57 (1944), 385-430.

3. I. P. Natanson, Konstruktive Funktionentheorie, Akademie-Verlag, Berlin, 1955.

4. A. Zygmund, Trigonometric series, Cambridge Univ. Press, Cambridge, 1959.

University of California, Los Angeles 